\title{
Mutational analysis of TSC1 and TSC2 genes in Japanese patients with tuberous sclerosis complex
}

\begin{abstract}
We have surveyed the mutations of TSC1 and TSC2 from 38 (25 sporadic, 11 familial, and 2 unknown) Japanese patients with tuberous sclerosis complex. In 23 of 38 subjects, we detected 18 new mutations in addition to 4 mutations that had been previously reported. We also found 3 new polymorphisms. The mutations were not clustered on a particular exon in either of the genes. Seven TSC1 mutations found in 3 familial and 4 sporadic cases were on the exons ( 3 missense, 2 nonsense point mutations, a 1-base insertion, and a 2-bp deletion). Fifteen TSC2 mutations were found in 5 familial cases, 10 sporadic cases, and 1 unknown case. The 12 mutations were on the exons $(8$ missense, 1 nonsense point mutations, a 1-bp insertion, a 5bp deletion, and a 4-bp replacement) and 3 point mutations were on the exon-intron junctions. Although the patients with TSC2 mutations tend to exhibit relatively severe mental retardation in comparison to those with TSC1 mutations, a genotype-phenotype correlation could not yet be established. The widespread distribution of TSC1/TSC2 mutations hinders the development of a simple diagnostic test, and the identification of individual mutations does not provide the prediction of prognosis.
\end{abstract}

Key words Tuberous sclerosis complex $\cdot T S C 1$ gene $\cdot T S C 2$ gene $\cdot$ Hamartin $\cdot$ Tuberin $\cdot$ Mutation

H. Zhang $\cdot$ E. Nanba $(\bowtie) \cdot T$. Yamamoto

Gene Research Center, Tottori University, Nishimachi 86, Yonago 683-8503, Japan

Tel. +81-859-34-8283; Fax +81-859-34-8284

e-mail: enanba@grape.med.tottori-u.ac.jp

H. Zhang $\cdot$ H. Ninomiya $\cdot K$. Ohno

Department of Neurobiology, School of Life Science, Faculty of

Medicine, Tottori University Yonago, Japan

K. Takeshita

Division of Child Neurology, Institute of Neurological Sciences,

Faculty of Medicine, Tottori University, Yonago, Japan

M. Mizuguchi

Department of Pediatrics, Jichi Medical School, Tochigi, Japan

\section{Introduction}

Tuberous sclerosis complex (TSC) is an autosomal dominant disease with an estimated prevalence of 1 in 10,000 in the Caucasian population and 1 in 31,000 in the Japanese population (Ohno et al. 1981; Gomez 1988). Multiple organs are affected in TSC, including the central nervous system (mental retardation, epilepsy), skin (facial angiofibroma, shagreen patches, hypopigmented macules, periungual fibromas), heart (cardiac rhabdomyomas), and kidney (cysts, angiomyolipoma).

There are two causative genes of TSC. The TSC1 (hamartin) is located on $9 \mathrm{q} 34$ and consists of 23 exons. The TSC2 (tuberin) is located on 16p13.3 and consists of 41 exons (European Chromosome 16 Tuberous Sclerosis Consortium 1993; Van Slegtenhorst et al. 1997). Loss of heterozygosity (LOH) of either TSC1 or TSC2 in the affected tissues indicates that both act as a tumor suppressor (Green et al. 1994; Henske et al. 1995, 1996; Sepp et al. 1996). Multiple isotypes of tuberin are generated by alternative splicing at two different exons (exon 25 and 31) of TSC2 (Xiao et al. 1997). Since the identification of the TSC1/ TSC2 genes, the mutations in Caucasian TSC patients have been extensively surveyed (Kumar et al. 1995 a,b; Vrtel et al. 1996; Wilson et al. 1996; Bajek et al. 1997; Jones et al. 1997; Maheshwar et al. 1997; Van Slegtenhorst et al. 1997; Au et al. 1997, 1998). The mutations in Japanese TSC patients, however, have remained unknown.

In the present study, we surveyed the mutations in Japanese patients and assessed the relationship between the mutations and clinical symptoms.

\section{Materials and methods}

Patients and sample collection

Thirty-eight ( 25 sporadic, 11 familial, and 2 unknown) Japanese TSC patients were clinically diagnosed according to the criteria (Gomez 1988). In some cases, ultrasound renogra- 
phy, MRI, and CT brain scan gave supportive evidence for the diagnosis. Genomic DNAs were prepared from the blood samples as described (Nanba et al. 1995). Healthy Japanese volunteer DNA as normal controls were extracted by the Nucleic Acid Purification System (MagExtractor MFX-2000; Toyobo, Osaka, Japan) from $100 \mu$ l blood.

Polymerase chain reaction-single strand conformation polymorphism (PCR-SSCP) analysis

All coding exons of TSC1 and TSC2 were analyzed for every patient. Sets of PCR primers for the 41 exons of TSC2 were designed based on the TSC2 sequence, except for the primers for exon 13 and 25, which were kindly obtained by Dr. J.R. Sampson at the University of Wales, Cardiff, UK (Maheshwar et al. 1996) (Table 1). The primer information for the 21 coding exons of the TSC1 gene was obtained from Dr. O Hino in the Department of Experimental Pathology,
Cancer Institute, Tokyo, Japan, and Dr. D.J. Kwiatkowski in Brigham and Womens' Hospital, Boston, USA, except for the primers for exon 9 (CACTGAGTTGACACTCTGAAG and CTGAACTAAGTCTTACTCCAG), which were originally designed. PCR contained $25 \mathrm{ng}$ of the genomic DNA as a template, $10 \mathrm{pmol}$ of each primer, $0.25 \mu \mathrm{M}$ of each dNTP, 0.1 unit of Ampli Taq Gold (Perkin-Elmer, Norwalk, CT, USA), and a buffer in a total volume of $5 \mu \mathrm{l}$. Cycling conditions were as follows: an initial denaturation at $95^{\circ} \mathrm{C}$ for $10 \mathrm{~min}$ followed by 30 cycles of denaturation at $95^{\circ} \mathrm{C}$ for $1 \mathrm{~min}$, annealing at $65^{\circ} \mathrm{C}$ for $1 \mathrm{~min}$ and elongation at $72^{\circ} \mathrm{C}$ for $1 \mathrm{~min}$, with a final extension at $72^{\circ} \mathrm{C}$ for $5 \mathrm{~min}$. All the PCR products were analyzed on $1.5 \%$ agarose gel. SSCP analysis was performed as described (Orita et al. 1989; Yuasa et al. 1997). Using a minigel $(10 \times 10 \mathrm{~cm})$, the samples were analyzed under four different electrophoresis conditions, in a combination of two sets of gel mixtures (12\% polyacrylamide gel with or without $5 \%$ glycerol) and two temperature conditions $\left(4^{\circ} \mathrm{C}\right.$ or $\left.22^{\circ} \mathrm{C}\right)$.

Table 1. Primers for $T S C 2$ gene analysis

\begin{tabular}{|c|c|c|}
\hline \multicolumn{2}{|l|}{ Exon } & \multirow{2}{*}{$\frac{\text { Product size (bp) }}{296}$} \\
\hline 1 & F: AAGGTTATGCCCACCAGAGA; R: AGACACAGGTAGCTCACTCA & \\
\hline 2 & F: CACTGGCCCCTTTTTCTTCT; R: CTCTAGTAGCTCAACTGGAT & 150 \\
\hline 3 & F: CTTGGAGAGCACATCCTCAC; R: CAGCCTGACGTCACCCATCC & 195 \\
\hline 4 & F: CTGATCCTGTGGCTTTTGTC; R: AGAAACCTCCAACCCAAGGT & 199 \\
\hline 5 & F: TCGCAAACTGCGCGACTTCT; R: CAGAACAGGATCTCAATTCT & 173 \\
\hline 6 & F: GACTGAGCTCGGTGCTCCCT; R: TGAAATGGGCCGGCCCTGGG & 109 \\
\hline 7 & F: GAAGGAGGTGGGAAGGAAG; R: CATCCATGTGCTCTCAGGAA & 278 \\
\hline 8 & F: CTTTGGGAGGAGATGGTGG; R: ATGTGTGCGTTGGTAAGGGA & 286 \\
\hline 9 & F: CGTCTCTCTGGGGAACACTT; R: TCACTGCACACAGAAACCGC & 176 \\
\hline 10 & F: TGCTGGCCGGGCTCGTGTTC; R: TGCTCCCCGGAGCTCCTGCC & 198 \\
\hline 11 & F: GCCTGTGTCATCGTGCCTGG; R: AAAGGCCCCACCCAGGAGGG & 194 \\
\hline 12 & F: CAACACCGGCTCTTCTTTTG; R: AGGCAAGGCTACAGAGGACC & 153 \\
\hline $13^{\mathrm{a}}$ & F: GGGCTGTGGCGGGCACTC; R: AGAGGTCCGGCAGACCGAAGTC & 245 \\
\hline 14 & F: TTGGCCTCCCTTGTGCCTGT; R: GCCCCGGGTACAACGGCTCT & 203 \\
\hline 15 & F: GACTCAGAACCATGAGCCTG; R: AACAGACTCCAACACAACGC & 251 \\
\hline 16 & F: CGTGGTGAGCTGCGTCCTCT; R: ACTGCGTGCGCAACCCCAGC & 178 \\
\hline 17 & F: GGCTTTCACCATCCTCTTCC; R: TCATGGGCCGTGGGCAGGTC & 160 \\
\hline 18 & F: GCCTCAGCTGCTTCTCTTGC; R: AACGTGGGACGGATGGTCCC & 216 \\
\hline 19 & F: CCTGACGCCTCСТCТCCTCG; R: CCCAACCCCAGGCCGGCATG & 185 \\
\hline 20 & F: TCATGCCTGGATTTGGTCAT; R: TCAAGCTCGCCTGCTCTGAC & 187 \\
\hline 21 & F: GGGCCTGAGGTGTCCTGTCT; R: AGGTGCAGTGCTGCAGGGCG & 246 \\
\hline 22 & F: GGCTCCCTGACCACCTCTCC; R: ACAGGGCCTGGGGCGACCAC & 148 \\
\hline 23 & F: GTGCCAGCCCCCTTCTCATC; R: ACAGCCTTCACCGCCCTGAG & 152 \\
\hline 24 & F: TCACTGTCTGGGTGTGCTC; R: TGCCCTGCCTCACATACTC & 260 \\
\hline $25^{\mathrm{a}}$ & F: CCCTCCACTGGCTTGTTCTCC; R: CGGGCAAGACGATGAGGTCAT & $-^{\mathrm{b}}$ \\
\hline 26 & F: CCTGGTCACGGCCTCTCCCT; R: CCAGCCCTGTAGTGCCGCCT & 213 \\
\hline 27 & F: GGTTTCACGCTCCCTGTCTT; R: GGAGGAAAGGAAGGTGCAGT & 200 \\
\hline 28 & F: ACCCTGTGCGTGGGATTCTC; R: AGGTGGCTGGGGCCAAGGCT & 163 \\
\hline 29 & F: CCTCATGCCCGTCTTCTTC; R: AGATGGCGCTCAGGCCAC & 267 \\
\hline 30 & F: AAGGTAGTCTGCCGCCTCCG; R: CCAAGGCCCGCCATGCCACT & 251 \\
\hline 31 & F: CGTCGACACGGCCTTCCCTT; R: ACCGCTTCCCTCTGAGAGGC & 118 \\
\hline 32 & F: AGCAGCCCCGTCTGTGTCCT; R: GTCCAGGCTCTGAGCCACAC & 171 \\
\hline 33 & 1F: CTCTTTGGGATGGTCCTTTC; 1R: CTGTGACCGGGCCTTAACCT & 275 \\
\hline 33 & 2F: CCTGGGGACAAGGCCGACGT; 2R: CCCGCCCGGAAGCAAGAGGC & 317 \\
\hline 34 & F: CCACCATCCCCTCCCTGT; R: TAGCCAGAGCCCTCTGCAA & 206 \\
\hline 35 & F: CTCTGGCTAAGCTCCCTGT; R: TGAGCACTTCATGCTGTAGG & 326 \\
\hline 36 & F: GGGAGTGATGCCACCCTG; R: ATGGGAGCCGTGCCCTGA & - \\
\hline 37 & F: CATCCGGCCCTGCTCACCCT; R: GCCTCACTGACGGCCCTCAC & 187 \\
\hline 38 & F: TGACCCTTTCTCTTGTCCGG; R: TCTGCACTGCAGCCCCACCC & 131 \\
\hline 39,40 & F: TGGCGTGACCACCAAGTCTC; R: GCTGAGGAGCCCCATATTCC & 325 \\
\hline 41 & F: CAGACTTACTGCCCAAGCCGCCT; R: CCCCGCACCAAGCAGACAAA & 342 \\
\hline
\end{tabular}

${ }^{a}$ Data were kindly provided by Dr. J.R. Sampson (University of Wales, UK)

${ }^{\mathrm{b}}$ The exact sizes of the PCR products were not known 
Sequencing

Variant bands were reamplified and subcloned into pGEM$\mathrm{T}$ Vector (Promega, Madison, WI, USA). The DNA sequences were determined using the Thermo Sequenase Fluorescent Labeled Primer Cycle Sequencing Kit (Amersham, Buckinghamshire, England) with Cy-5-labeled M13 universal or reversal primers and ALFred Automatic Sequencer (Pharmacia Biotech, Tokyo, Japan). At least ten independent clones of each PCR product were sequenced in both directions. The sequence data were analyzed by Genetyx computer software (Software Development, Tokyo, Japan).

\section{Results}

\section{SSCP analysis}

A total of 25 variants were detected by SSCP analysis (Fig. 1) from 23 patients $(60 \%$ of the total 38 patients). Seven variants of TSC1 gene were detected in 3 familial cases and 4 sporadic ones. Fifteen variants of TSC2 gene were detected in 5 familial cases, 10 sporadic cases, and one unknown case. L574L of TSC1 and I531I of TSC2 genes were considered to be polymorphisms because the point mutations did not cause amino acid substitution. M322T of TSC1 was detected in the normal controls (6 of 80$)$ and was supposed to represent a polymorphism (Table $2 \mathrm{~A}$ ). All other missense mutations were not detected in 60 or 100 normal controls. Any DNA sample from the parents was not available except for P28.

\section{Mutations in TSC1 gene}

Two nonsense, two frame shift, and three missense mutations were located in various exons of the gene. Two mutations (R509X, Q654E) in three patients were located in exon 15, and the R509X was found both in a sporadic patient (P26) and a familial patient (P3). Two mutations (W676X and T417I) were found in one patient (P31).

\section{Mutations in TSC2 gene}

One nonsense, 3 frame shift, and 8 missense mutations were identified in 16 patients (Table $2 \mathrm{~B}$ ). The two single nucleotide substitutions $(2760+1 \mathrm{G}$ to A, $5179-1 \mathrm{G}$ to $\mathrm{T})$ of an exon-intron junction were predicted to cause a splicing error. Although a substitution $(5178+5 \mathrm{G}$ to $\mathrm{A})$ in the intron was not in the consensus sequence of the splicing, the mutation was located $5 \mathrm{bp}$ distant from the exon and was not detected in 100 normal controls. The mutation might cause a splicing error. L717R in a patient with pulmonary tuberous sclerosis was reported previously (Zhan et al. 1999). Both the V769E mutation (P2 and P21) and R1459X mutation (P6 and P8) were detected in 2 unrelated sporadic patients. Two missense mutations (H137R and D647N)
TSC 1

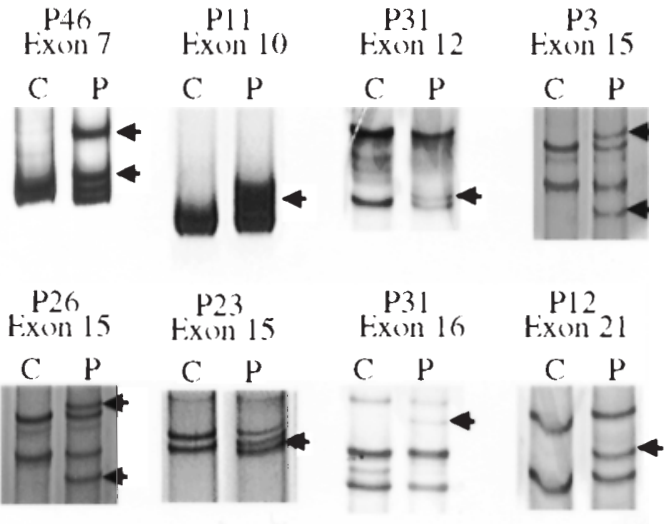

\section{TSC2}
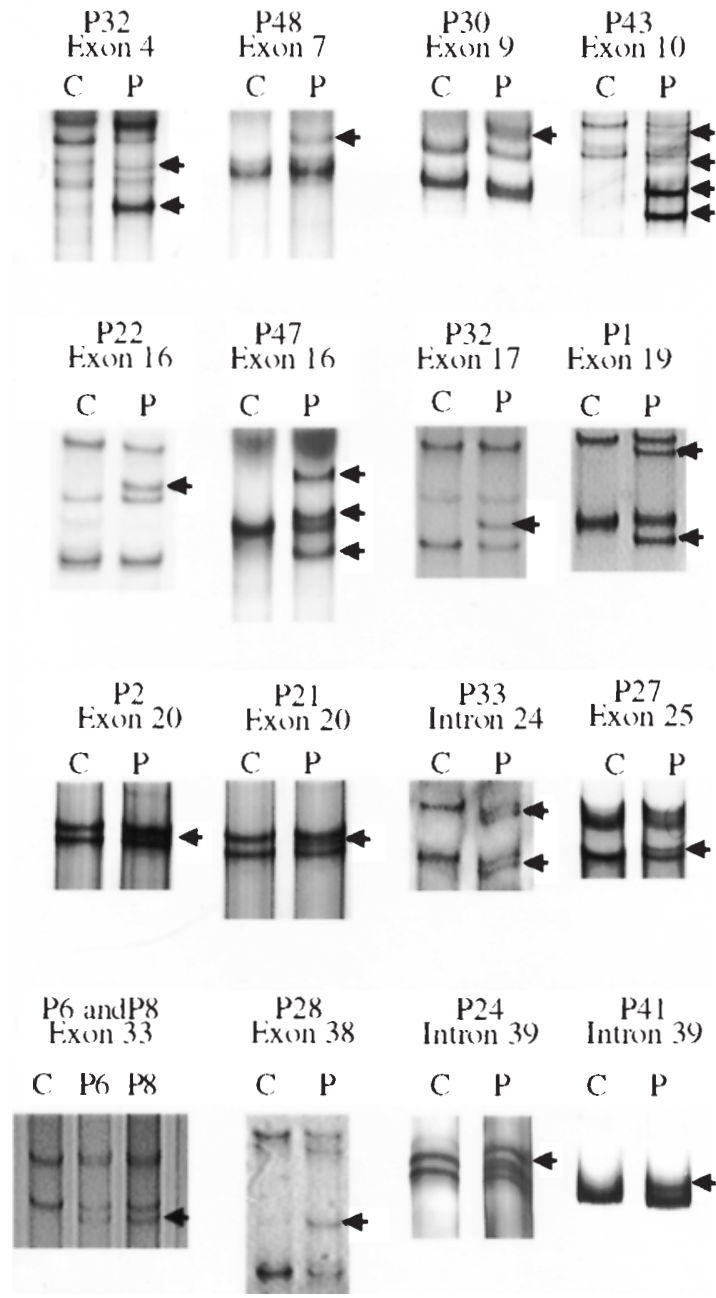

C P $\quad \mathrm{C} \quad \mathrm{P}$

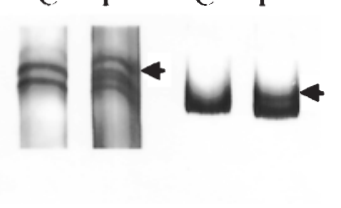

Fig. 1. PCR-SSCP analysis of the TSC1/TSC2 genes. Arrows indicate the aberrant bands found in the patients' samples. $C$, control samples from healthy subjects; $P$, patients' samples 
Table 2A. Mutations in TSC1

\begin{tabular}{llllll}
\hline Patient & Amino acid change & Location & $\begin{array}{l}\text { Type of } \\
\text { mutation }\end{array}$ & $\begin{array}{l}\text { Nucleotide } \\
\text { alteration }\end{array}$ & S/F* \\
\hline 46 & 216PheFS-216X(a) & exon 7 & Frame shift & 866delTT & $\mathrm{F}$ \\
11 & 311SerFS-340X & exon 10 & Frame shift & 1210insT & F \\
31 & T4171 & exon 12 & Missense & 1471 CtoT & S \\
26,3 & R509X(b) & exon 15 & Nonsense & 1746 CtoT & F,S \\
23 & Q654E(b) & exon 15 & Missense & 2181 CtoG & S \\
31 & W676X & exon 16 & Nonsense & 2249 GtoA & S \\
12 & T899S & exon 21 & Missense & 2918 CtoG & S \\
$46,27,3$ & L574L & exon 15 & Polymorphism & 1947 TtoC & \\
$17,34,36$ & M322T & exon 10 & Polymorphism & 1186 TtoC & \\
\hline
\end{tabular}

Table 2B. Mutations in TSC2

\begin{tabular}{llllll}
\hline Patient & Mutation & Location & $\begin{array}{l}\text { Type of } \\
\text { mutation }\end{array}$ & $\begin{array}{l}\text { Nucleotide } \\
\text { alteration }\end{array}$ & S/F* \\
\hline 32 & H137R & Exon 4 & Missense & 428 AtoG & S \\
48 & 238LeuFS-337X & Exon 7 & Frame shift & 725 insT \\
30 & F320L & Exon 9 & Missense & 978 TtoG & S \\
43 & 337GluFS-351X & Exon 10 & Frame shift & 1029GATCdel, CATGGCAT ins & F \\
22 & R611Q(c) & Exon 16 & Missense & 1850 GtoA & F \\
47 & 597HisFS-614X & Exon 16 & Frame shift & 1808delACTAC & S \\
32 & D647N & Exon 17 & Missense & 1957 AtoG & S \\
1 & L717R & Exon 19 & Missense & 2168 TtoG & S \\
21,2 & V769E & Exon 20 & Missense & 2324 TtoA & F,S \\
33 & - & Intron 24 & Splicing & $2760+1$ GtoA & S \\
27 & V963M & Exon 25 & Missense & 2905 GtoA & S,S \\
6,8 & R1459X & Exon 33 & Nonsense & 4393 CtoT & S \\
28 & P1657L(d) & Exon 38 & Missense & 5042 CtoT & F \\
24 & - & Intron 39 & Splicing & $5178+5$ GtoA & F \\
41 & - & Intron 39 & Splicing & $5179-1$ GtoT & \\
33 & 15311 & Exon 14 & Polymorphism & 1611 CtoT & \\
\hline
\end{tabular}

*S, sporadic; $\mathrm{F}$, familial

The initiator ATG begins at base 222 of TSC1 and at base 19 for TSC2

The original amino acid and its position in the protein (numbered from the initiator Met as 1) are followed by a new amino acid for missense mutations, and by $\mathrm{X}$ for nonsense mutation

${ }^{a, b}$ The identical change was reported by Van Slegtenhorst et al. (1997)

${ }^{\mathrm{c}}$ The identical change was reported by Wilson et al. (1996)

${ }^{\mathrm{d}}$ The identical change was reported by Maheshwar et al. (1997)

were found in the same patient (P32). P1657L in P28 was confirmed to be a de novo mutation by examination of the parents' samples.

\section{Clinical symptoms and mutation types}

The clinical symptoms and the gene mutations are summarized in Table 3 . The number of the patients with mental retardation $(+$ and ++$)$ in TSC2 was significantly larger than that in TSC1 $\left(\chi^{2}=4.8, P=0.0285\right)$. The severity of mental retardation of the patients with TSC1 mutations appeared to be milder than that of the patients with $T S C 2$ mutations. Complete information on renal and cardiac involvements was not available and could not be assessed. None of the mutation types (missense, nonsense, frame shift, and splicing) appeared to be correlated with a specific clinical symptom. In particular, the R509X mutation in TSC1 was detected in two patients, and the severity of mental retardation in these patients were quite different.

\section{Discussion}

Mutations in a given gene may be screened by various methods such as Southern blot analysis, SSCP, heteroduplex analysis, protein truncation test, and RT-PCR followed by SSCP (Kumar et al. 1995a,b; Vrtel et al. 1996; Wilson et al. 1996; Bajek et al. 1997; Jones et al. 1997; Maheshwar et al. 1997; Au et al. 1997, 1998). In the present study, we employed SSCP to survey the mutations of TSC1 and TSC2 genes because the method is simple enough to cover the multiple numbers of exons. To increase the mutation detection rate of SSCP, we used four electrophoresis conditions by a combination of different sets of temperature and glycerol content of the gel. Our mutation detection rate was $60 \%$ (23 of 38), which is comparable with those in other reports (Jones et al. 1997; Van Slegtenhorst et al. 1997; Au et al. 1998).

There are several possible explanations for the failure to detect of TSC1/TSC2 mutations in the 15 patients. First, it can be false negative. One report showed that the SSCP 
Table 3. Clinical data of mutation detected patients

\begin{tabular}{|c|c|c|c|c|c|c|c|c|c|c|}
\hline $\begin{array}{l}\text { Patient } \\
\text { number }\end{array}$ & Age & Sex & $\begin{array}{l}\text { Mental } \\
\text { retardation }\end{array}$ & $\mathrm{AF}$ & $\begin{array}{l}\text { White } \\
\text { macules }\end{array}$ & $\begin{array}{l}\text { Subependymal } \\
\text { nodules }\end{array}$ & Seizures & $\mathrm{S} / \mathrm{F}$ & Mutations & Location of mutation \\
\hline 46 & 9 & $\mathrm{M}$ & + & - & + & + & + & $\mathrm{F}$ & Frame shift & TSC1 exon 7 \\
\hline 11 & 7 & $\mathrm{~F}$ & \pm & + & + & + & + & $\mathrm{F}$ & Frame shift & TSC1 exon 10 \\
\hline 31 & 8 & $\mathrm{~F}$ & + & n.d. & + & + & + & S & Missense/nonsense & TSC1 exon 12; TSC1 exon 16 \\
\hline 3 & 32 & $\mathrm{~F}$ & ++ & n.d. & n.d. & n.d. & n.d. & $\mathrm{S}$ & Nonsense & TSC1 exon 15 \\
\hline 26 & 21 & M & - & - & + & + & - & $\mathrm{F}$ & Nonsense & TSC1 exon 15 \\
\hline 23 & 21 & M & + & - & + & + & + & S & Missense & TSC1 exon 15 \\
\hline 12 & 32 & $\mathrm{~F}$ & - & + & + & + & + & $\mathrm{S}$ & Missense & TSC1 exon 21 \\
\hline 32 & 2 & M & n.d. & n.d. & + & + & + & S & Missenses & TSC2 exon 4; TSC2 exon 17 \\
\hline 48 & 24 & M & ++ & + & + & + & + & $\mathrm{S}$ & Frame shift & TSC 2 exon 7 \\
\hline 30 & nd & nd & n.d. & n.d. & n.d. & n.d. & n.d. & n.d. & Missense & TSC2 exon 9 \\
\hline 43 & 8 & M & ++ & + & + & + & + & $\mathrm{F}$ & Frame shift & TSC 2 exon 10 \\
\hline 22 & 6 & $\mathrm{~F}$ & - & - & + & + & + & $\mathrm{F}$ & Missense & TSC2 exon 16 \\
\hline 47 & 6 & $\mathrm{~F}$ & + & + & + & + & + & S & Frame shift & TSC2 exon 16 \\
\hline 1 & 17 & $\mathrm{~F}$ & - & + & + & + & + & $\mathrm{S}$ & Missense & TSC 2 exon 19 \\
\hline 21 & 44 & $\mathrm{~F}$ & ++ & + & + & n.d. & + & $\mathrm{F}$ & Missense & TSC 2 exon 20 \\
\hline 2 & 32 & M & ++ & + & + & + & + & S & Missense & TSC2 exon 20 \\
\hline 33 & 48 & $\mathrm{M}$ & ++ & n.d. & n.d. & n.d. & + & $\mathrm{S}$ & Splicing & TSC2 intron 24 \\
\hline 27 & 13 & M & - & - & + & + & + & $\mathrm{F}$ & Missense & TSC2 exon 25 \\
\hline 6 & 8 & M & ++ & n.d. & n.d. & n.d. & n.d. & $\mathrm{F}$ & Nonsense & TSC2 exon 33 \\
\hline 8 & 9 & $\mathrm{~F}$ & ++ & + & + & + & + & $\mathrm{S}$ & Nonsense & TSC 2 exon 33 \\
\hline 28 & 3 & $\mathrm{~F}$ & + & n.d. & + & + & + & S & Missense & TSC2 exon 38 \\
\hline 24 & 12 & $\mathrm{M}$ & - & - & + & + & + & $\mathrm{F}$ & Splicing & TSC2 intron 39 \\
\hline 41 & 7 & M & - & + & + & + & + & $\mathrm{F}$ & Splicing & TSC2 intron 39 \\
\hline
\end{tabular}

n.d., information not available; $\mathrm{AF}$, angiofibroma; S, sporadic; F, familial

method to detect a point mutation on a 250-bp DNA fragment was $63 \%$ effective (Sheffield et al. 1993). Second, it may be true negative in those cases in which the mutation lies outside of the coding sequence, either in the $5^{\prime}$-regulatory elements, within the intronic sequences, or in the 3'UTR. In addition, a large-scale rearrangement of genomic DNA may yield true negative results in the SSCP of the coding exons. Although there is no report of a large-scale mutation in TSC1, there are indeed some in TSC2 (Jones et al. 1997; The European Chromosome 16 Tuberous Sclerosis Consortium 1993; Au et al. 1997; Van Slegtenhorst et al. 1997).

The mutation surveys of TSC1/TSC2 genes in Caucasian TSC patients revealed no clustering of the mutation in either gene (Wilson et al. 1996; Jones et al. 1997; Van Slegtenhorst et al. 1997; Au et al. 1998). In accordance with these previous findings, the 22 mutations (18 new and 4 reported) found in this study were distributed on various exons and there was no clustering of the mutations. Again in accordance with the previous findings, there was no obvious relationship between the type and location of the mutation and the clinical symptoms. However, the patients with TSC2 mutations appeared to have more severe mental retardation compared with the patients with TSC1 mutations. The same tendency has been noted (Jones et al. 1997). It is therefore at least clear that the clinical phenotype cannot be predicted from the genotype. Because of the heterogeneity of the mutations, it also appears impossible at present to develop a simple diagnostic test for TSC.

Acknowledgements We thank Dr. Satoko Kumada, Dr. Akira Uchiyama (Tokyo Metropolitan Fuchu Ryoiku Center), Dr. Youich Sakakihara, Dr. Hidekazu Takeshita (Departement of Pediatrics, Graduate School of Medicine, University of Tokyo), Dr. Khotaro
Izumi, Dr. Masahiro Nakagawa (Third Department of Internal Medicine, Faculty of Medicine, Kagoshima University), Dr. Ryutaro Kira (Department of Pediatrics, Kyushu University), Dr. Hitoshi Sejima (Department of Pediatrics, Shimane Medical University), Dr. Masahito Miyazaki (Department of Pediatrics, School of Medicine, Tokushima University), Dr. Akira Kuramochi (Department of Dermatology, Saitama Medical School), and Dr. Sadahisa Ohta (Department of Neurosurgery, Tokyo Medical and Dental University) for collecting the samples of the TSC patients. This work was supported by the Ministry of Health and Welfare of Japan by a special grant for neurocutaneous disease, and by the Ministry of Education, Science, Sport and Culture of Japan by a grant-in-aid for scientific research.

\section{References}

Au KS, Rodriguez JA, Rodriguez E, Robyns WB, Delgado MR, Northrup H (1997) Mutations and polymorphisms in the tuberous sclerosis complex gene on chromosome 16. Hum Mutat 9:23-29

Au KS, Rodriguez JA, Finch JL, Volcik KA, Roach ES, Delgado MR, Rodriguez E, Northrup H (1998) Germ-line mutational analysis of the TSC2 gene in 90 tuberous-sclerosis patients. Am J Hum Genet 62:286-294

Bajek IV, Sepp T, Ward S, Yates JRW, Green AJ (1997) Mutations in the TSC2 gene: analysis of the complete coding sequence using the protein truncation test (PTT). Hum Mol Genet 6:1406-1414

European Chromosome 16 Tuberous Sclerosis Consortium (1993) Identification and characterization of the tuberous sclerosis gene on chromosome 16. Cell 75:1305-1315

Gomez M (1988) Tuberous sclerosis. Raven Press, New York

Green AJ, Smith M, Yates JR (1994) Loss of heterozygosity on chromosome 16p13.3 in hamartomas from tuberous sclerosis patients. Nat Genet 6:193-196

Henske EP, Neumann HP, Scheithauer BW, Herbst EW, Short MP, Kwiatkowski DJ (1995) Loss of heterozygosity in the tuberous sclerosis (TSC2) region of chromosome band 16p13 occurs in sporadic as well as TSC-associated renal angiomyolipomas. Genes Chromosomes Cancer 13:295-298

Henske EP, Scheithauer BW, Short MP, Wollmann R, Nahmias J, Hornigold N, Vanslegtenhorst M, Welsh CT, Kwiatkowski DJ 
(1996) Allelic loss is frequent in tuberous sclerosis kidney lesions but rare in brain lesions. Am J Hum Genet 59:400-406

Jones AC, Daniells CE, Snell RG, Tachataki M, Idziaszczyk SA, Krawczak M, Sampson JR, Cheadle JP (1997) Molecular genetic and phenotypic analysis reveals differences between TSC1 and TSC2 associated familial and sporalic tuberous sclerosis. Hum Mol Genet 6:2155-2161

Kumar A, Kandt RS, Wolpert C, Roses AD, Pericak-Vance MA, Gilbert JR (1995a) Mutation analysis of the TSC2 gene in an African-American family. Hum Mol Genet 4:2295-2298

Kumar A, Wolpert C, Kandt RS, Segal J, Pufky J, Roses AD, PericakVance MA, Gilbert JR (1995b) A de novo frame-shift mutation in the tuberin gene. Hum Mol Genet 4:1471-1472

Maheshwar MM, Sandford R, Nellist M, Cheadle JP, Sgotto B, Vaudin M, Sampson JR (1996) Comparative analysis and genomic structure of the tuberous sclerosis 2 (TSC2) gene in human and pufferfish. Hum Mol Genet 5:131-137

Maheshwar MN, Cheadle JP, Jones AC, Myring J, Fryer AE, Harris PC, Sampson JR (1997) The GAP-related domain of tuberin, the product of the TSC2 gene, is a target for missense mutations in tuberous sclerosis. Hum Mol Genet 6:1991-1996

Nanba E, Kohno Y, Matsuda A, Yano M, Sato C, Hashimoto K, Koeda T, Yoshino K, Kimura M, Maeoka Y, Yamamoto T, Maegaki Y, Eda I, Takeshita K (1995) Non-radioactive DNA diagnosis for the fragile $X$ syndrome in mentally retarded Japanese males. Brain Dev 17:317-321

Ohno K, Takeshita K, Arima M (1981) Frequency of tuberous sclerosis in San-in district (Japan) and birth weight of patients with tuberous sclerosis. Brain Dev 3:57-64

Orita M, Iwahana H, Kanazawa H, Hayashi K, Sekiya T (1989) Detection of polymorphisms of human DNA by gel electrophoresis as single-strand conformation polymorphisms. Proc Natl Acad Sci USA 86:2766-2770

Sepp T, Yates JRW, Green AJ (1996) Loss of heterozygosity in tuberous sclerosis hamartomas. J Med Genet 33:962-964
Sheffield VC, Blck JS, Kwitek AE, Sandstrom DW, Stone EM (1993) The sensitivity of single-strand conformation polymorphism analysis for the detection of single base substitutions. Genomics 16:325-332

Van Slegtenhorst M, Dehoogt R, Hermans C, Nellist M, Janssen B, Verhoef S, Lindhout D, Vandenouweland A, Halley D, Young J, Burley M, Jeremiah S, Woodward K, Hahamias J, Fox M, Ekong R, Osborne J, Wolfe J, Povey S, Snell RG, Cheadle JP, Jones AC, Tachataki M, Ravine D, Sampson JR, Reeve MP, Prchrdon P, Wilmer F, Munro C, Hawkins TL, Sepp T, Ali JBL, Ward S, Green AJ, Yates JRW, Kwiatkowska J, Henske EP, Short MP, Haines JH, Jozwiak S, Kwiatkowski DJ (1997) Identification of the tuberous sclerosis gene TSC1 on chromosome 9q34. Science 277:805-808

Vrtel R, Verhoef S, Bouman K, Maheshwar MM, Nellist M, Vanessen AJ, Bakker P, Hermans CJ, Binkboelkens M, Vanelburg RM, Hoff M, Lindhout D, Sampson J, Halley D, Vandenouweland A (1996) Identification of a nonsense mutation at the $5^{\prime}$ end of the TSC 2 gene in a family with a presumptive diagnosis of tuberous sclerosis complex. J Med Genet 33:47-51

Wilson P, Ramesh V, Kristiansen A, Bove C, Jozwiak S, Kwiatkowski D, Short M, Haines J (1996) Novel mutations detected in the TSC2 gene from both sporadic and familial TSC patients. Hum Mol Genet 5:249-256

Xiao GH, Shoarinejad F, Jin F, Golemis EA, Yeung RS (1997) The tuberous sclerosis 2 gene product, tuberin, functions as a Rab5 GTPase activating protein (GAP) in modulating endocytosis. J Biol Chem 272:6097-6100

Yuasa I, Umetsu K, Vogt U, Nakamura H, Nanba E, Tamaki N, Irizawa Y (1997) Human orosomucoid polymorphism: molecular basis of the three common ORM1 alleles, ORM1*F1, ORM1*F2, and ORM1*S. Hum Genet 99:393-398

Zhan H, Yamamoto T, Nanba E, Kitamura Y, Terada T, Akaboshi S, Yuasa I, Ohtani K, Nakamoto S, Takeshita K, Ohno K (1999) Novel TSC2 mutation in a patient with pulmonary tuberous sclerosis: lack of loss of heterozygosity in a lung cyst. Am J Med Genet 82:368-370 